This is the peer reviewed version of the following article:

J.-A. Amalian, S. Poyer, B. E. Petit, S. Telitel, V. Monnier, D. Karamessini, D.Gigmes, J.-F.

Lutz, L.Charles

Adv. Funct. Mater. 2017, 27, 1604595,

which has been published in final form at

https://doi.org/10.1016/j.ijms.2017.07.006.

This article may be used for non-commercial purposes in accordance with Elsevier Terms and Conditions."

\title{
Negative mode MS/MS to read digital information encoded in sequence-defined oligo(urethane)s: a mechanistic study
}

Jean-Arthur Amalian, ${ }^{1}$ Salomé Poyer, ${ }^{1}$ Benoit Eric Petit, ${ }^{2}$ Sofia Telitel, ${ }^{2}$ Valérie Monnier, ${ }^{3}$ Denise Karamessini, ${ }^{2}$ Didier Gigmes, ${ }^{1}$ Jean-François Lutz, ${ }^{2 *}$ Laurence Charles ${ }^{1 *}$

${ }^{1}$ Aix Marseille Univ, CNRS, ICR, Institut de Chimie Radicalaire, Marseille - France, ${ }^{2}$ Université de Strasbourg, CNRS, Institut Charles Sadron UPR22, 23 rue du Loess, 67034 Strasbourg Cedex 2, France, jflutz@unistra.fr., ${ }^{3}$ Aix Marseille Univ, CNRS, Fédération des Sciences Chimiques de Marseille, FR 1739, Marseille-France, laurence.charles@univ-amu.fr

ABSTRACT: MS/MS sequencing is an unrivaled technique to decipher binary information chemically encoded in the backbone of sequence-controlled synthetic polymers constructed with two co-monomers of different mass, arbitrarily designated as the 0- and 1-bit of the ASCII alphabet. Efficiency of this "reading" step relies however on the simplicity of MS/MS patterns, which depends on both polymer chemistry and chain length. In this context, polyurethanes (PUs) were very promising candidates as dissociation of small deprotonated oligomers $(\mathrm{n}<8)$ yielded a single fragment series. The carbamate bond cleavage reaction was hence studied in details to tentatively anticipate the CID behavior of longer chains prior to optimizing their synthesis. In spite of the simplicity of MS/MS spectra, three different mechanisms were evidenced; however, they were not expected to induce MS/MS complexity when activating longer chains, as verified or sequence-controlled PUs containing up to two bytes of information (i.e., 16 co-monomers). In contrast, the ionization step appeared to be an issue: deprotonation yield of the end-group in negative ion mode electrospray was observed to strongly decrease as PU chain length increases. This sensitivity issue was addressed by introducing a second acidic end-group to allow doubly deprotonated oligomers with no impact on their CID behavior. 


\section{Introduction}

Synthetic digital polymers have recently emerged as an interesting new class of macromolecules that can be used to store information at the molecular level [1,2]. By using a set of two co-monomers arbitrarily defined as the 0- and 1-bits of the ASCII alphabet [3,4], our group has reported various synthesis methodologies involving different chemistries to produce so-called digitally encoded polymers such as poly(alkoxyamine amide)s[5,6], poly(phosphodiester)s [7,8], poly(alkoxyamine phospho-diester)s [9], poly(triazole amide)s [10,11], and polyurethanes[12,13]. In all these species, the chemical code was based on mass, with a simple $\mathrm{H} / \mathrm{CH} 3$ molecular variation between the two co-monomers. As a result, MS/MS sequencing appeared as a key technique to "read" information that was "written" in the chains. Indeed, similarly to biopolymers such as peptides [14], oligonucleotides [15], or carbohydrates [16], collision-induced dissociation (CID) of sequence-defined synthetic polymers proceeds via back-bone cleavages independently of comonomer arrangement along the chain, yielding MS/MS data that allow their sequence to be reconstructed. However, complexity of CID spectra (which affects MS/MS readability of encoded information) highly depends on the chemistry of the polymer backbone, as commonly observed for synthetic polymers [17]. Most often, and similar to the case of biopolymers, multiple fragment series are generated upon activation, which leads to redundant information when used for sequencing purposes. Moreover, spectral complexity further increases with the size of dissociating chains: because longer chains promptly adopt high charge states upon electrospray ionization(ESI), their dissociation products also exhibit a charge state distribution. Typically, a chain-length of at least 100 residues is wished for such digital polymers, in order to prepare libraries of moderate size macromolecules to compose long messages rather than using very high molecular weight polymers, as learnt from studies involving DNA chains as a medium to store information [18,19].In this context, simplicity of MS/MS pattern obtained for small oligomers (n $<10)$ prepared in the earlier development stages of new sequence-defined polymers is a key requirement before dedicating further efforts in optimizing synthesis protocols to produce longer congeners with perfect monodispersity. So far, candidates that best fulfilled this requirement were polyurethanes (PUs) [12].As depicted in Scheme 1, information-encoded PUs are built from two co-monomeric units composed of a carbamate function linked to an alkyl segment, the latter being used to implement a binary $0 / 1$ code with $\mathrm{R}=\mathrm{H}$ for the 0 -bit and $\mathrm{R}=\mathrm{CH} 3$ for the 1-bit. Cooligomeric chains are capped by a carboxypentyl-end-group and a hydroxyl-termination. Although their structure allowed coded PU oligomers to readily ionize in both polarity modes, dissociation of deprotonated molecules was found to offer an extremely simple MS/MS pattern, with a single series of fragments spaced by the mass of one or the other co-monomer (vide infra). This peculiar pattern allows any binary messages to be literally read from CID spectra. In order to figure out whether such a simple MS/MS sequencing could also be anticipated for longer chains, mechanisms underlying dissociation reactions observed for PU co-oligomers were studied in details. 


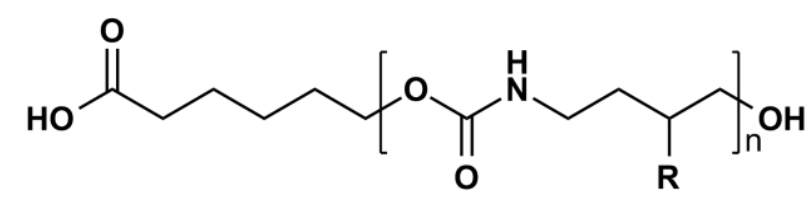

Scheme 1. Structure of information-containing oligourethane with a defined sequence of $\mathbf{0}\left(\mathrm{R}=\mathrm{H}, \mathrm{mo}_{0}=115.1 \mathrm{Da}\right)$ and $\mathbf{1}\left(\mathrm{R}=\mathrm{CH}_{3}, \mathrm{~m}_{1}=129.1 \mathrm{Da}\right)$ co-monomeric units.

\section{Experimental}

\subsection{Chemicals}

Digitally-encoded polyurethanes studied in this work were synthesized using an orthogonal iterative solid-phase approach described in a recent publication [12]. The oligourethane with ancarboxylic acid moiety was prepared using ethyl 6-aminohexanoate in the last step of the iterative synthesis. The terminal ester function was then acidified during cleavage and the formed ethanol was evaporated along with cleavage reagents, thus affording the desired compound. The sample containing a brominated end-group was prepared using a reported post-oligomerization modification procedure [20].All oligomer samples (a few $\mathrm{mg}$ ) were first solubilized in methanol (SDS, Peypin, France), then diluted $\left(1 / 10^{3}-1 / 10^{4}\right.$, v/v)in a methanolic solution of ammonium acetate $(3 \mathrm{mM})$ purchased from Sigma Aldrich (Saint Louis, MO), prior being subjected to electrospray ionization (ESI). Poly(ethylene glycol) (PEG) and poly(methylmethacrylate) (PMMA) used as internal standards for accurate mass measurements were from Sigma Aldrich. Deuterated methanol (CD3OD, 99.80\%D) used for H/D exchange experiments was from Eurisotop (Saint-Aubin, France). In these experiments, a $50 \mu \mathrm{L}$ sample solution was evaporated to dryness and dissolved in $100 \mu \mathrm{L} \mathrm{CD3OD} \mathrm{(3} \mathrm{times)} \mathrm{prior} \mathrm{injection} \mathrm{in} \mathrm{the} \mathrm{ESI} \mathrm{source.}$

\subsection{Mass spectrometry}

High resolution MS and MS/MS experiments were performed using a QStar Elite mass spectrometer (Applied Biosystems SCIEX, Concord, ON, Canada). Ions were accurately mass measured after internal calibration of the orthogonal acceleration time-of-flight (oa-TOF) mass analyzer, using two anionic adducts of PEG or PMMA to bracket the targeted analyte $\mathrm{m} / \mathrm{z}$ value in the MS mode [21] or the precursor ion signal in the MS/MS mode. In CID experiments, precursor ions were selected in a quadrupole mass analyzer, injected into the collision cell, and product ions were measured in the oa-TOF. Low resolution MS3experiments were performed usinga3200 QTRAP mass spectrometer (Applied Biosystems SCIEX).This instrument is equipped with two quadrupole mass analyzers separated by a collision cell, hence allowing classical MS/MS experiments. However, applying appropriate voltages at both the entrance and the exit of the second mass analyzer allows this quadrupole to be operated as a linear ion trap. As a result, MS3experiments can also be performed, with the first dissociation stage occurring in the collision cell while the second one takes place in the linear ion trap. Both instruments were equipped with an electrospray ionization source operated in the negative ion mode(capillary voltage: $-4200 \mathrm{~V}$; cone voltage: $-75 \mathrm{~V})$, using air as the nebulizing gas (10 psi) and nitrogen as the curtain gas (20 psi) and as the collision gas. Sample solutions were introduced in the ESI source with a syringe 
pump at a $5 \mu \mathrm{L} \min ^{-1}$ flow rate. Instrument control, data acquisition and data processing of all experiments were achieved using Analyst software (QS 2.0 for QStar, 1.6.3 forQ-TRAP) provided by Applied Biosystems SCIEX.A Waters Synapt G2 HDMS mass spectrometer was used to perform pseudo-MS3experiments, also known as post-IMS parallelMS3-type acquisition. Typically, the quadrupole mass analyzer was used for selection of precursor ions that were further activated in an ion trap (collision energy: $35 \mathrm{~V}$; argon pressure: $0.5 \mathrm{bar}$ ). So-obtained product ions were then all ejected from the trap, passed through a cooling cell (helium flow: $180 \mathrm{~mL} \mathrm{~min}^{-1}$ ) and reached the Travelling Wave Ion Mobility Spectrometry (TWIMS) cell operated at 2.94 mbar of $\mathrm{N} 2$, with a wave velocity $\left(\mathrm{m} \mathrm{s}^{-1}\right)$ /wave height (V) couple of 1100/40. After IMS separation, fragments were activated in a collision cell (collision energy: $25 \mathrm{~V}$; argon pressure: 0.5 bar) and ions generated during this second activation stage were mass measured using the oa-TOF mass analyzer in the $50-1500 \mathrm{~m} / \mathrm{z}$ range. The ESI source of this instrument was operated in the negative ion mode (capillary voltage: $-2.2 \mathrm{kV}$; sampling cone voltage: $-50 \mathrm{~V}$; desolvation gas (N2) flow: $300 \mathrm{~L} \mathrm{~h}^{-1}$ at $150^{\circ} \mathrm{C}$; source temperature: $50^{\circ} \mathrm{C}$ ). Instrument control, data acquisition and data processing of all experiments were achieved with the MassLynx4.1 programs, while 2D-plots were generated with Driftscope 2.1, both provided by Waters.

\section{Results and discussion}

Sequence-defined PUs were ionized in the negative ion mode ESI after deprotonation of the carboxylic acid group in the $\alpha$ moiety (Scheme 1). Single stage MS experiments confirmed that the requested uniform structure has been achieved for these sequence-defined polymers, with main detection of targeted species validating that all chains exhibit the same length (Sup-porting Fig. S1). As mentioned in the Introduction, MS/MS spectra obtained for deprotonated PU oligomers were extremely simple and hence readily allowed full coverage of their sequence, as described in details hereafter.

\subsection{CID of deprotonated PUs}

Collisional activation of deprotonated PU oligomers generated a single fragment series, with the distance between two consecutive congeners being equal to the mass of one or the other comonomeric unit [12]. Such CID spectra easily allowed reading of any message in digitally encoded PUs, as exemplified with the two isomeric chains 101010 (Fig. 1a) and 000111 (Fig. 1b) of the same co-monomeric composition but distinct sequences. This very simple dissociation pattern resulted from the occurrence of a single bond cleavage in each repeating unit, that is, the $\mathrm{O}(\mathrm{CO})$ linkage of the carbamate moiety, combined with the fact that the negative charge remained located at the $\alpha$ chain end, hence preventing any complementary products to be detected. 

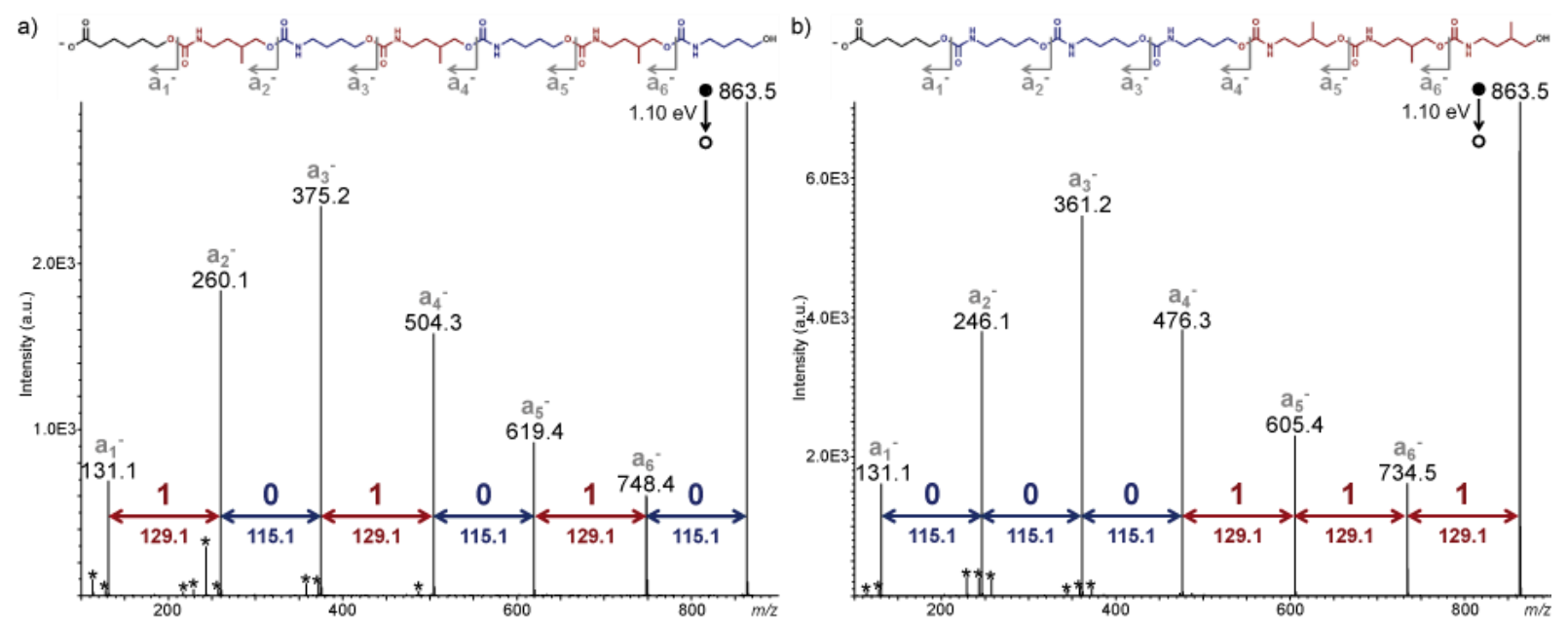

Fig. 1.Negative mode ESI-MS/MS recorded at $1.10 \mathrm{eV}$ (center-of-mass frame) for a)101010 and b) $000111 \mathrm{PU}$ oligomers, both as deprotonated molecules $(\mathrm{m} / \mathrm{z}$ 863.5), with corresponding fragmentation scheme on top. * designates secondary fragments.

This greatly contrasts with the complex dissociation behavior of protonated PUs formed in positive mode ESI, observed to yield four ion series together with abundant secondary fragments (Sup-porting Fig. S2), as previously reported for related species [22,23]. According to the nomenclature established for synthetic polymer fragments [17], product ions generated from deprotonated Pus were named $\mathrm{a}_{\mathrm{i}}{ }^{-}$as they were formed upon cleavage of the first bond in the monomeric unit, with the subscripted i designating the total number of partial or entire repeating units they contain. Owing to their $\mathrm{m} / \mathrm{z}$ values and as supported by accurate mass measurements (Supporting Table S1), $\mathrm{a}_{\mathrm{i}}^{-}$fragments were formed after a proton has been transferred from the righthand side of the dissociating center to the ether oxygen atom of the carbamate moiety. In order to find out which proton was transferred and whether $\mathrm{a}_{\mathrm{i}}{ }^{-}$fragments were formed according to competitive or consecutive processes, this reaction was mechanistically studied using the 101010 oligomer as a model.

\subsection{Competitive vs consecutive mechanisms.}

Both consecutive and competitive processes can be envisaged to account for the $\mathrm{O}(\mathrm{CO})$ bond cleavage in the carbamate moiety. As previously reported during CID of ester-based polyurethanes in the positive ion mode [23], proton transfer can proceed via a charge-remote reaction from the nearby amide NH group. Accordingly, $a_{i}^{-}$fragments could be formed either from the precursor ion (in a competitive manner) or upon dissociation of higher $\mathrm{a}_{i}^{-}$congeners (in a consecutive manner). The alternative proton transfer proceeding from the terminal $\mathrm{OH}$ can also be envisaged to occur consecutively and competitively. However, the efficiency of the latter process is expected to decrease when considering formation of small $\mathrm{a}_{\mathrm{i}}{ }^{-}$ions that would imply a long distance proton transfer. Monitoring the abundance of $a_{i}{ }^{-}$ions as a function of the activation energy imparted to their precursor (Fig. 2a) confirmed that both competitive and consecutive processes were at work. 
On the one hand, a similar energy threshold of about $0.60 \mathrm{eV}$ was measured for all product ions (except a1-), hence in favor of competitive processes. On the other hand, activation energy allowing maximum fragment abundance (highlighted by a square in each data series of Fig. 2a) was noticed to increase as the fragment size decreased, as typically observed for consecutive processes. The smallest $a_{i}{ }^{-}$congener at $\mathrm{m} / \mathrm{z} 131.1$ was a noticeable exception, as its detection required significantly higher activation energy (at least $0.85 \mathrm{eV}$, center-of-mass frame) for the $\mathrm{m} / z$ 863.5 precursor (red plot in Fig. 2a). This particular behavior suggested that a1-was formed according to a different reaction as compared to larger fragments.
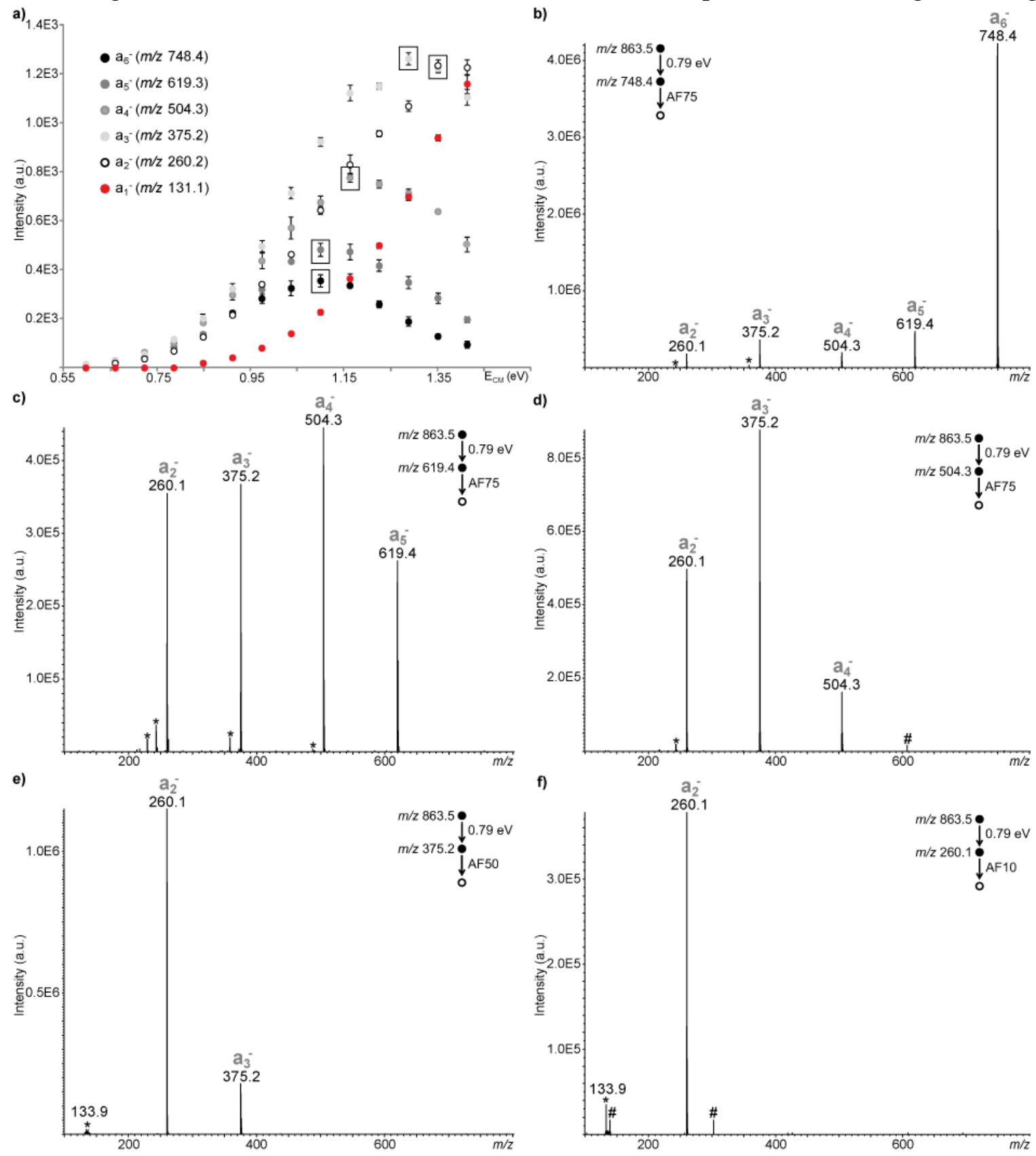
Fig. 2. a) Breakdown curves of $\mathrm{a}_{\mathrm{i}}^{-}$fragments from $[101010-\mathrm{H}]^{-}$at $m / z$ 863.5. $\mathrm{MS}^{3}$ spectra obtained after activation of b) $\mathrm{a}_{6}{ }^{-}$at $m / 2748.4$, c) $\mathrm{a}_{5}{ }^{-}$at $m / z 619.3$, d) $\mathrm{a}_{4}{ }^{-}$at $m / z 504.3$, e) $\mathrm{a}_{3}{ }^{-}$at $\mathrm{m} / \mathrm{z} 375.2$,and f) $\mathrm{a}_{2}{ }^{-}$at $\mathrm{m} / \mathrm{z} 260.2 .{ }^{*}$ designates secondary fragments and \# electronic noise.

Data recorded in MS3experiments (Fig. 2b-f) confirmed these assumptions. On the one hand, all $\mathrm{a}_{\mathrm{i}}^{-}$fragments (with $\mathrm{i}=2-5$ ) of the deprotonated 101010 6-mer were formed upon activation of any $a_{n}{ }^{-}$ions (with $n>i$ ), hence in a consecutive manner. On the other hand, the smallest al-ion was never observed in any of theseMS3spectra, indicating that cleavage of the first carbamate bond only proceeded from the precursor ion. Alternatively, this last result could be explained by the fact that energy imparted to ions in the linear ion trap during the second activation stage might not be high enough to induce this particular reaction.

\subsection{Mechanism for $\mathrm{O}(\mathrm{CO})$ bond cleavage in carbamate functions}

H/D exchange (HDX) experiments were performed in order to identify which proton transfer induced the $\mathrm{O}(\mathrm{CO})$ bond cleavage in carbamate moieties. When prepared in CD3OD, the studied101010 6-mer was detected as a deprotonated molecule with mostly up to $6 \mathrm{D}$ atoms at $\mathrm{m} / \mathrm{z}$ 869.5, while full H/D exchange con-ducting to a $7 \mathrm{D}$ containing species was detected to a small extent at $m / z 870.5$ (Supporting Fig. S3a). Similarly, HDX experiments con-ducted for different PU molecules systematically showed their $\mathrm{m} / z$ values to mostly shift by a number equal to their polymerization degree (data not shown). Although surprising, this result suggested that H/D exchanges mainly occurred in all carbamate groups but hardly in the hydroxyl end-group. This assumption was further supported by HDX data obtained for PU oligomers with an ester moiety instead of $\mathrm{OH}$ as the $\omega$ termination: as shown in Supporting Fig. S3b, m/z value measured for the ester derivative 4-mer 0001clearly indicated deuteration of the four carbamate groups. CID data recorded for the 101010-d6 oligomer at m/z 869.5 are presented in Fig. 3. It should be noted that, to perform HDX experiments, no ammonium acetate was added in PU samples prior to ESI in order to avoid H/D back exchanges in solution. As a result, depro-tonation yield of PUs was poor, and the number of accumulated spectra had to be increased by a factor of 10 in MS/MS to compensate for the quite low absolute abundance of product ions. In this MS/MS spectrum, two peaks were observed for all $\mathrm{a}_{\mathrm{i}}{ }^{-}$ions, hence formed after transfer of either $\mathrm{D}(\mathrm{m} / \mathrm{z}$ in pink) or $\mathrm{H}(\mathrm{m} / \mathrm{z}$ in purple) according to mechanisms proposed in Scheme 2a-b for cleavage of the $\mathrm{O}(\mathrm{CO})$ bond. Variation of relative abundance of these two peaks as a function of the fragment size showed that formation of product ions via $\mathrm{H}$-transfer became less favored as their size decreased. This result suggests that $\mathrm{H}$-transfer mostly occurred from the terminal group (Scheme $2 b$ ): this would be consistent with the lower efficiency expected for such long range proton transfers and would further support that the 101010-d6 precursor ion mostly contained an OH (instead of OD) terminal group. 


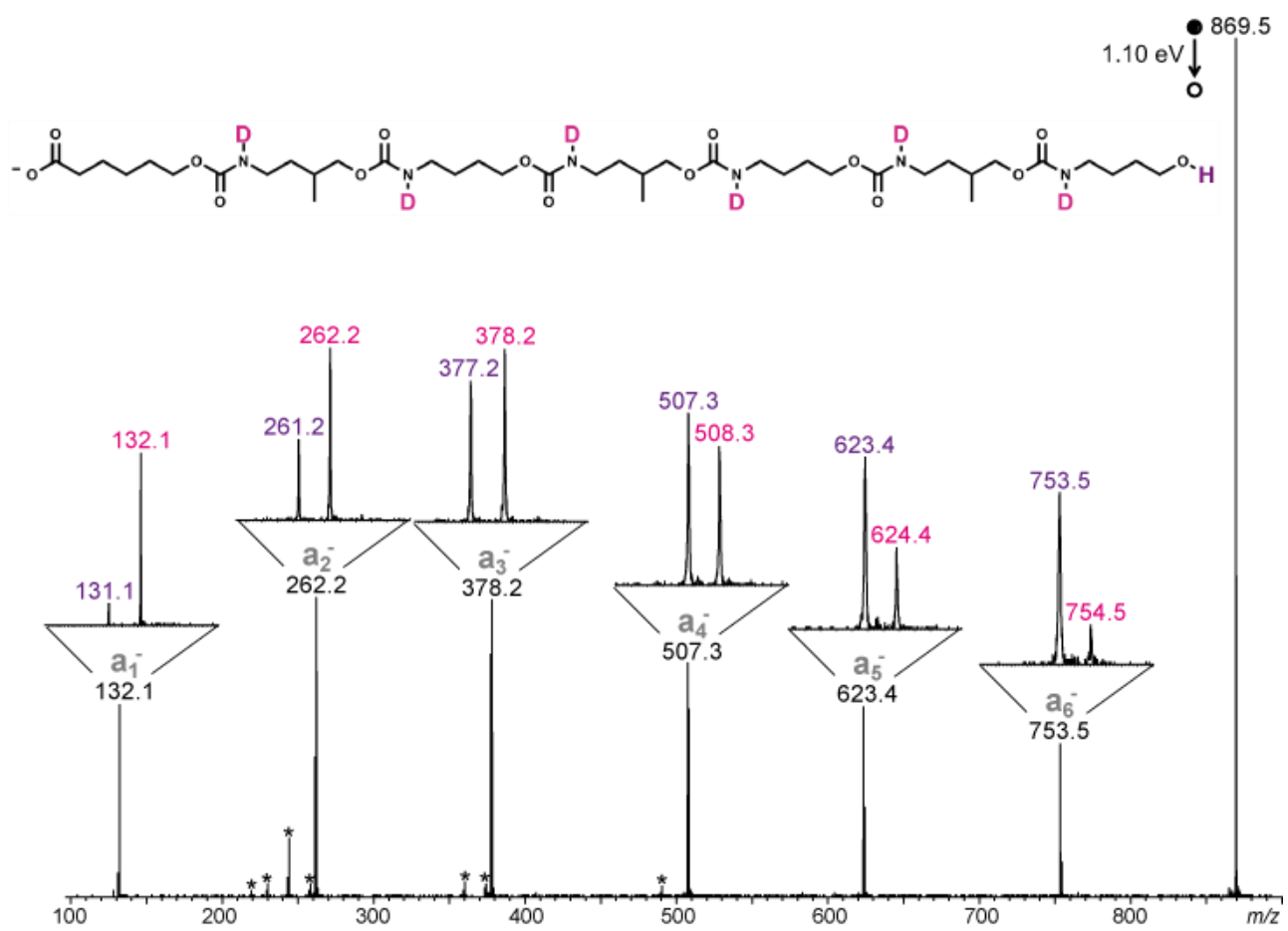

Fig. 3. Negative mode ESI-MS/MS of the 101010-d6 oligomer (shown in inset) at $\mathrm{m} / z$ 869.5. Colored $m / z$ values indicate which $\mathrm{a}_{\mathrm{i}}{ }^{-}$form was generated after transfer of $\mathrm{D}$ in carbamate moieties (pink) or $\mathrm{H}$ from hydroxy group (purple). * designates secondary fragments.

a)<smiles>[R]C1CCNC(=O)C(C([R])CCNC(=O)OCC([R])CCNC(=O)OCCCCCC(=O)OC)OCC1</smiles><smiles>[R]C(CCN=C=O)C([R])CCNC(=O)OC(C)(C)CCCCC(=O)OC</smiles>

b)

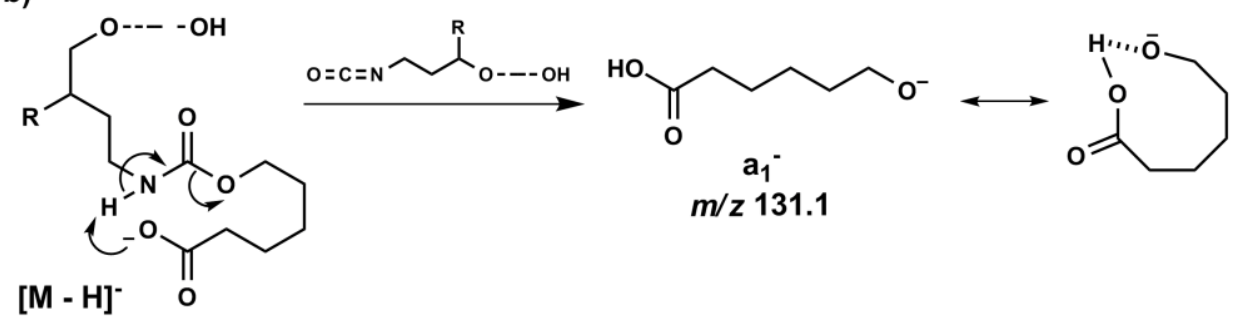

Scheme 2.a) Proton transfer from carbamate NH (plain arrows) or terminal OH (dotted arrows) to account for the formation of $a_{i}{ }^{-}$fragments with $i>1$. b) Alternative mechanism proposed for the specific formation of $\mathrm{a}_{\mathrm{i}}^{-}$at $\mathrm{m} / \mathrm{z} 131.1$. 
To account for the more energy demanding reaction leading to $\mathrm{a}_{1}{ }^{-}$, a particular conformation of the $\alpha$ termination was proposed (Scheme 2c), in which folding of the pentyl segment would allow the carboxylate moiety to strongly interact with the $\mathrm{NH}$ proton of the first unit. As a result, this proton could no longer be transferred to induce the cleavage of the nearby $\mathrm{O}(\mathrm{CO})$ bond according to the pathway shown in Scheme 2a. Alternatively, activation of the pre-cursor ion would lead to protonation of the carboxylate group, and subsequent electron transfers would conduct to the formation of $\mathrm{a}_{1}{ }^{-}$(Scheme 2c). This mechanism is consistent with CID of 101010-d6 showing a preferential form of $\mathrm{a}_{1}{ }^{-}$at $\mathrm{m} / \mathrm{z}, 132.1$ as a result of deuterium transfer from the first carbamate moiety (Fig. 3). More-over, existence of this hydrogen bonded conformation might also prevent release of $\mathrm{CO} 2$, which was never observed from either the precursor or the product ions.

However, our assumption of too low activated $\mathrm{a}_{\mathrm{i}}{ }^{-}$fragments to account for the absence of $\mathrm{a}_{1}{ }^{-}$in all $\mathrm{MS}^{3}$ spectra of Fig. 3 (although these reactions could mechanistically be envisaged according to Scheme 2a) was still to be validated. For this purpose, pseudo-MS ${ }^{3}$ experiments were performed in a Synapt G2 instrument to achieve more energetic collisions during the second activation stage. In this post-IMS parallel $\mathrm{MS}^{3}$-type acquisition, the $\mathrm{m} / \mathrm{z} 863.5$ precursor ion was selected in a quadrupole mass analyzer and then dissociated in a quadrupole ion trap, while a collision cell was used to activate product ions that were separated in the mobility cell. Using constant instrumental settings for the second activation stage (i.e. $25 \mathrm{eV}$, laboratory frame) allowed the energy imparted to ai-fragments to increase as their size decreased, that is, $1.27 \mathrm{eV}$ for $m / z 748.4,1.51 \mathrm{eV}$ for $\mathrm{m} / z$ 619.4, $1.84 \mathrm{eV}$ for $\mathrm{m} / \mathrm{z} 504.3,2.41 \mathrm{eV}$ for $\mathrm{m} / \mathrm{z} 375.2$, and $3.33 \mathrm{eV}$ for $\mathrm{m} / \mathrm{z} 260.1$. In this collision regime, the a1-fragment could indeed be generated from all higher $\mathrm{a}_{\mathrm{i}}{ }^{-}$congeners, as clearly shown by signals measured for $m / z 131.1$ at all drift times of $\mathrm{a}_{\mathrm{i}}{ }^{-}$in the 2D-plot of Fig. 4. This result hence confirmed the limited activation energy available in the linear ion trap of the instrument used to acquire MS3data of Fig. 3. 


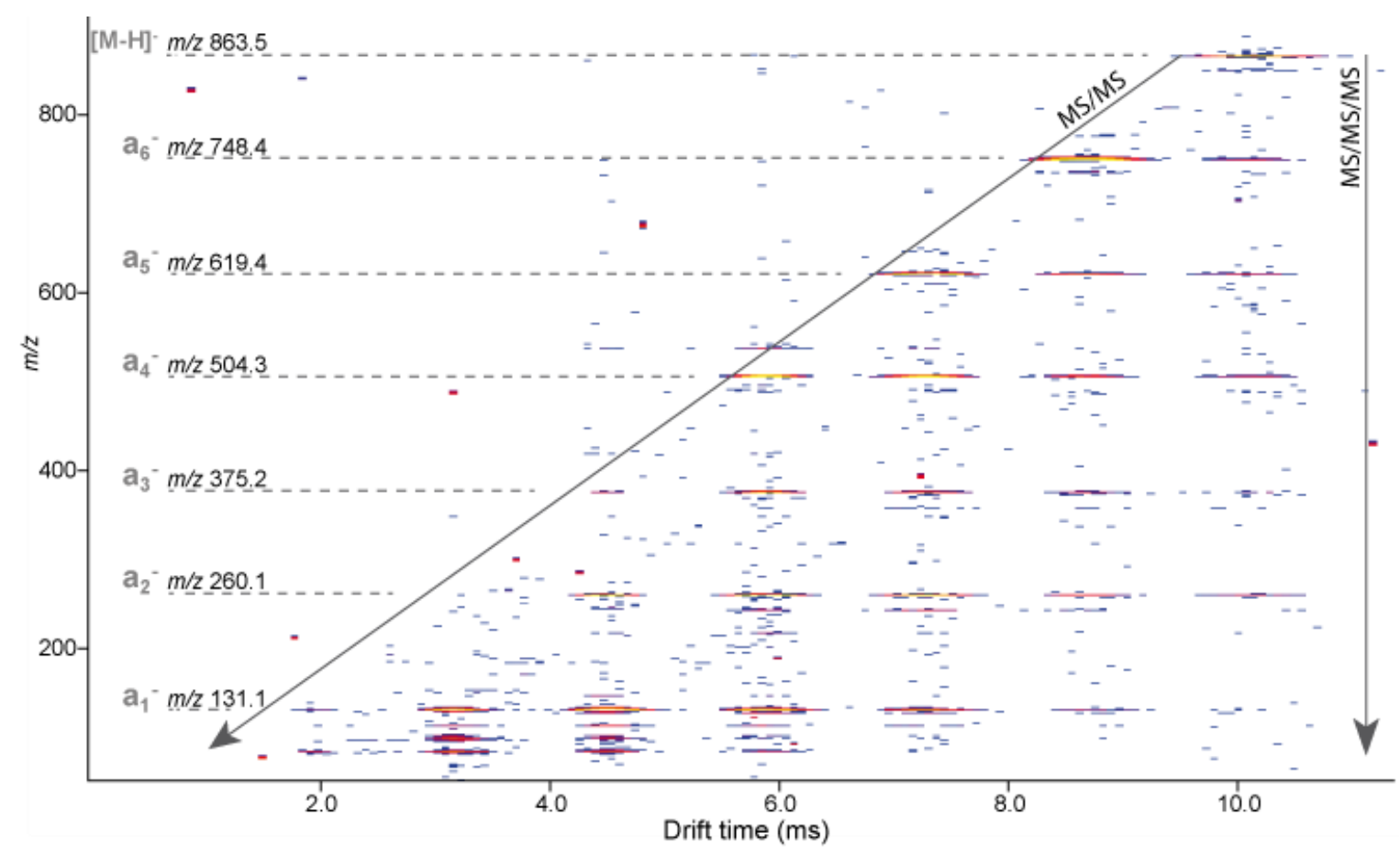

Fig. 4.Pseudo-MS ${ }^{3}$ data recorded on the Synapt G2 HDMS and represented as a 2D-plot, where fragments generated in the second activation stage are detected at the same drift time (hence vertically aligned) as compared to their respective precursor.

\subsection{CID of longer PU chains}

The PU synthesis protocol was then optimized to produce longer sequence-controlled chains. As illustrated for the 2-byte (i.e., 16 residues) PU coding for 1101001011011000 (Supporting Fig. S4a), monodispersity was achieved for this sample but the oligomer was detected as a trifluoroacetate adduct rather than a deprotonated molecule, indicating a strong decrease of deprotonation yield of the $\alpha$ group as the chain length increased. Nevertheless, activating this $[\mathrm{M}+\mathrm{TFA}]^{-}$gave rise to the elimination of one trifluoroacetic acid molecule to form $[\mathrm{M}-\mathrm{H}]^{-}$, which further dissociation yielded the same simple MS/MS pattern as smaller oligomers, with the whole $\mathrm{a}_{\mathrm{i}}^{-}$ion series allowing a full coverage of the digitally encoded PU sequence (Supporting Fig. S4b). Although not influencing the dissociation behavior of PUs, the chain length was observed to have a strong impact on sensitivity, due to a decreased ionization yield combined with a lower transmission efficiency of high $\mathrm{m} / \mathrm{z}$ ions by the quadrupole mass analyzer used for precursor selection in MS/MS. As a result, a large number of spectra had to be accumulated (50 in the MS mode, 500 in the MS/MS mode) to obtain data shown in Supporting Fig. S4.To address this issue, the $\omega$ termination was modified to include a second carboxylic acid function. Although operated on the small 00111 coded PU, this structural modification allowed doubly deprotonated molecules to be generated in the negative ion mode ESI. As illustrated in Fig. 5, this charge state increase did not modify the CID behavior of PU, with main fragmentation reactions still observed 
to be $\mathrm{O}-(\mathrm{CO})$ bond cleavage in each repeating unit. Due to both end-groups being deprotonated in these di-acidic species, a pair of complementary $\mathrm{a}_{\mathrm{i}}{ }^{-} / \mathrm{x}_{\mathrm{i}}{ }^{-}$fragments was detected upon cleavage at each carbamate moiety, allowing the 00111 sequence to be reconstructed from one or the other PU termination while not introducing too much complexity in the MS/MS data at least for the studied 5-mer. This derivatization protocol still needs to further be optimized to enable production of monodisperse samples of long sequence-controlled polymers.

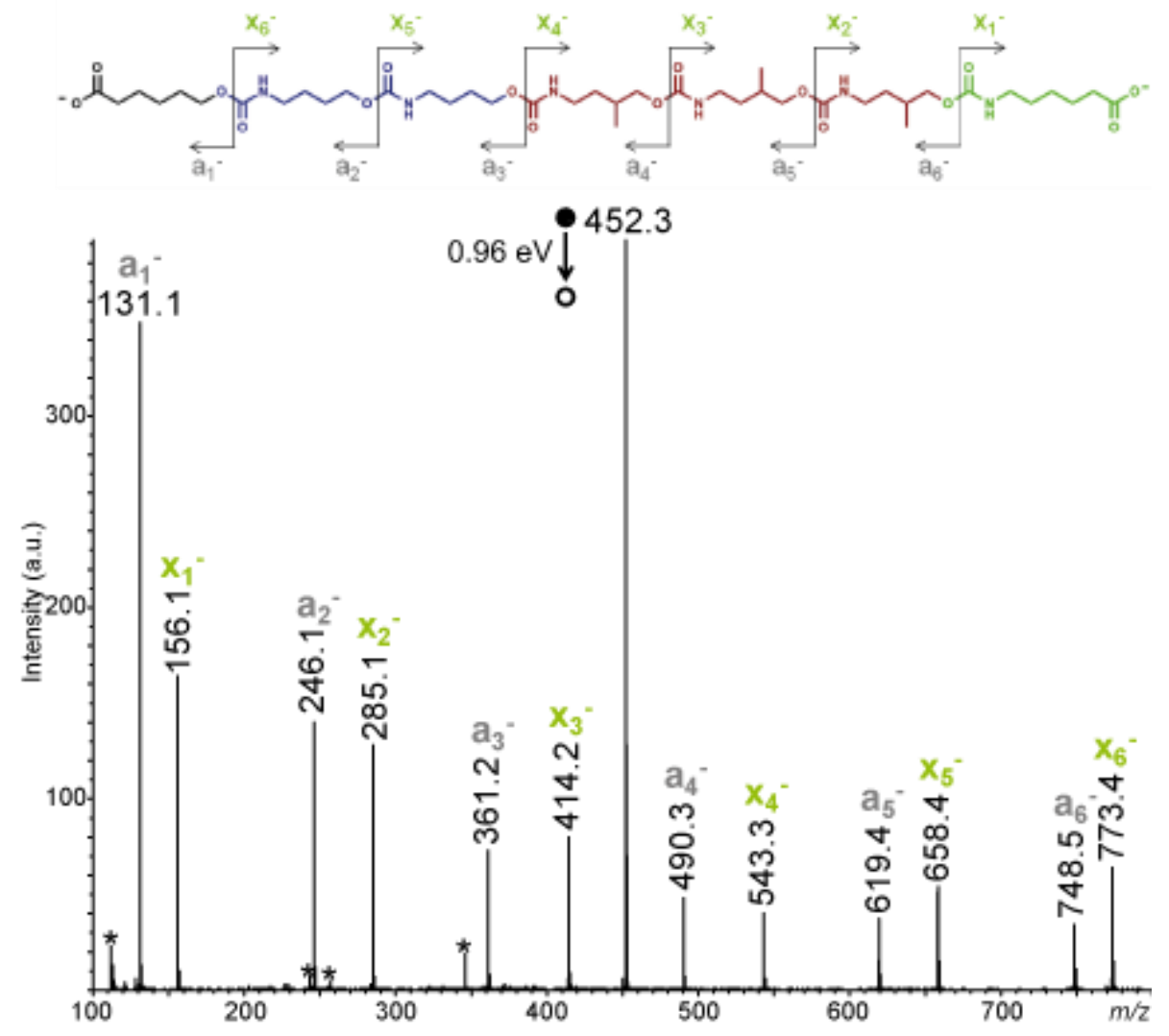

Fig. 5. Negative mode ESI-MS/MS $(0.96 \mathrm{eV}$, center-of-mass frame) of the di-acid terminated 00111 PU detected as a deprotonated molecule at $m / z$ 452.3. As indicated in the corresponding fragmentation scheme (top), fragments containing the original $\alpha$ moiety were named $\mathrm{a}_{\mathrm{i}}^{-}$(in grey) while ions containing the $\omega$ termination were named as $\mathrm{x}_{\mathrm{i}}^{-}$(in green). ${ }^{*}$ designates secondary fragments.

\section{Conclusion.}

As shown in this mechanistic study, most simple MS/MS pat-terns do not necessarily imply that dissociating ions experienced a single reaction. Cleavage of $\mathrm{O}(\mathrm{CO})$ bond in carbamate groups was indeed evidenced to proceed via three mechanisms of different efficiencies. The bottom-up approach implemented here to anticipate the CID behavior of long chains from that observed for very short oligomers showed the same dissociation reactions applied for Pus with a chain length up to 16 residues. However, this size limitation was not related to fragmentation issues but to a decreasing limit of detection of PUs as their size increased, due to a decreasing ionization yield 
combined to a lower transmission efficiency of high $\mathrm{m} / \mathrm{z}$ ions in the precursor ion selection stage. Introduction of a second acidic moiety to increase ion charge state (and hence decrease their $\mathrm{m} / \mathrm{z}$ ) was shown to be a promising strategy to address this issue, as this structural modification did not modify the CID behavior of digitally encoded PUs, as demonstrated for short oligomers. It should be acknowledged, however, that this approach will allow at most doubly charged species to be obtained: owing to the upper $\mathrm{m} / \mathrm{z}$ limit for precursor ion selection in the quadrupole analyzer used here, the upper limit to readable data size would be about 4 bytes of information. A more efficient strategy to store larger amounts of information in PU chains would consist of making use of language higher than binary.

Acknowledgments. L.C. acknowledges support from Spectropole, the Analytical Facility of Aix-Marseille University, by allowing a special access to the instruments purchased with European Funding (FEDEROBJ2142-3341). J.F.L. thanks the H2020 program of the European Union (project Euro-Sequences, H2020-MSCA-ITN-2014, grant agreement n॰642083), and the Cluster of Excellence Chemistry of Complex Systems (LabEx CSC) for financial support. The PhD positions of D. K. and B. E. P. are supported by the ITN Euro-Sequences and LabEX CSC, respectively.

Appendix A. Supplementary data associated with this article can be found, in the online version, at http://dx.doi.org/10.1016/j.ijms.2017.07.006.

\section{References:}

[1] J.-L. Lutz, M. Ouchi, D.R. Liu, M. Sawamoto, Sequence-controlled polymers, Science 341 (2013) 1238149.

[2] J.-F. Lutz, J.-M. Lehn, E.W. Meijer, K. Matyjaszewski, From precision polymers to complex materials and systems, Nat. Rev. Mater. 1 (2016) 16024.

[3] J.-F. Lutz, Writing on polymer chains, Acc. Chem. Res. 46 (2013) 2696-2705.

[4] H. Colquhoun, J.-F. Lutz, Information-containing macromolecules, Nat. Chem.6 (2014) 455-456.

[5] R.K. Roy, A. Meszynska, C. Laure, L. Charles, C. Verchin, J.-F. Lutz, Design and synthesis of digitally encoded polymers that can be decoded and erased, Nat.Commun.6 (2015) 7237.

[6] R.K. Roy, C. Laure, D. Fischer-Krauser, L. Charles, J.-F. Lutz, Convergent synthesis of digitally-encoded poly(alkoxyamine amide)s, Chem. Commun. 51(2015) 15677-15680.

[7] A. Al Ouahabi, L. Charles, J.-F. Lutz, Synthesis of non-natural sequence-encoded polymers using phosphoramidite chemistry, J. Am. Chem. Soc. 137 (2015) 5629-5635.

[8] A. Al Ouahabi, M. Kotera, L. Charles, J.-F. Lutz, Synthesis of monodisperse sequencecoded polymers with chain lengths above DP100, ACS Macro Lett. 4(2015) 1077-1080.

[9] G. Cavallo, A. Al Ouahabi, L. Oswald, L. Charles, J.-F. Lutz, Orthogonal synthesis of easyto-read information-containing polymers using phosphoramidite and radical coupling steps, J. Am. 
Chem. Soc. 138 (2016) 9417-9420.[10] T.T. Trinh, C. Laure, J.-F. Lutz, Synthesis of monodisperse sequence-defined polymers using protecting-group-free iterative strategies, Macromol. Chem.Phys. 216 (2015) 1498-1506.

[11] T.T. Trinh, L. Oswald, D. Chan-Seng, L. Charles, J.-F.Lutz, Preparation of informationcontaining macromolecules by ligation of dyad-encoded oligomers, Chem. Eur. J. 21 (2015) 11961-11965.

[12] U.S. Gunay, B.E. Petit, D. Karamessini, A. Al Ouahabi, J.-A. Amalian, C. Chendo,M. Bouquey, D. Gigmes, L. Charles, J.-F. Lutz, Chemoselective synthesis of uniform sequence-coded polyurethanes and their use as molecular tags, Chem 1 (2016) 114-126.

[13] D. Karamessini, B.E. Petit, M. Bouquey, L. Charles, J.-F. Lutz, Identification-tagging of methacrylate-based intraocular implants using sequence defined polyurethane barcodes, Adv. Funct. Mater.27 (2017)1604595.

[14] K. Biemann, Laying the ground work for proteomics - mass spectrometry from1958 to 1988, Int. J. Mass Spectrom. 259 (2007) 1-7.

[15] S.A. McLuckey, G.J. Vanberkel, G.L. Glish, Tandem mass-spectrometry of small:multiply charged oligonucleotides, J. Am. Soc. Mass Spectrom. 3 (1992) 60-70.

[16] B. Domon, C.E. Costello, A systematic nomenclature for carbohydrate fragmentations in FAB-MS/MS spectra of glycoconjugates, Glycoconjugate J. 5(1988) 397-409.

[17] C. Wesdemiotis, N. Solak, M.J. Polce, D.E. Dabney, K. Chaicharoen, B.C. Katzenmeyer, Fragmentation pathways of polymer ions, Mass Spectrom. Rev.30 (2011) 523-559.

[18] G.M. Church, Y. Gao, S. Kosuri, Next-generation digital information storage inDNA, Science 337 (2012), 1628-1628.

[19] N. Goldman, P. Bertone, S.Y. Chen, C. Dessimoz, E.M. LeProust, B. Sipos, E.Birney, Towards practical high-capacity, low-maintenance information storage in synthesized DNA, Nature 494 (2013) 77-80.

[20] S. Telitel, B.E. Petit, S. Poyer, L. Charles, J.-F. Lutz, Sequence-coded ATRP macroinitiators, Polym. Chem. (2017), http://dx.doi.org/10.1039/c7py00496f,in press.

[21] L. Charles, Influence of internal standard charge state on the accuracy of mass measurements in orthogonal acceleration time-of-flight mass spectrometers, Rapid Commun. Mass Spectrom. 22 (2008) 151-155.

[22] R. Warrass, P. Walden, K.H. Wiesmuller, G. Jung, Oligocarbamates as MHC class I ligands, Lett.Pept. Sci. 5 (1998) 125-128.

[23] A.P. Gies, D.M. Hercules, Collision induced dissociation study of ester-based polyurethane fragmentation reactions, Anal. Chim.Acta 808 (2014) 199-219. 